\title{
Diffusion Processes in the A-Model of Vector Admixture: Turbulent Prandtl Number
}

\author{
Eva Jurčišinová ${ }^{1,2, \star}$, Marián Jurčišin ${ }^{1,2,3, \star \star}$, and Richard Remecky $y^{1,2, \star \star \star}$ \\ ${ }^{1}$ Institute of Experimental Physics, SAS, Košice, Slovakia \\ ${ }^{2}$ Bogoliubov Laboratory of Theoretical Physics, JINR, Dubna, Russian Federation \\ ${ }^{3}$ Dep. of Theoretical Physics and Astrophysics, Faculty of Science, P.J. Šafárik University, Košice, Slovakia
}

\begin{abstract}
Using analytical approach of the field theoretic renormalization-group technique in two-loop approximation we model a fully developed turbulent system with vector characteristics driven by stochastic Navier-Stokes equation. The behaviour of the turbulent Prandtl number $\operatorname{Pr}_{\mathcal{A}, t}$ is investigated as a function of parameter $\mathcal{A}$ and spatial dimension $d>2$ for three cases, namely, kinematic MHD turbulence $(\mathcal{A}=1)$, the admixture of a vector impurity by the Navier-Stokes turbulent flow $(\mathcal{A}=0)$ and the model of linearized Navier-Stokes equation $(\mathcal{A}=-1)$. It is shown that for $\mathcal{A}=-1$ the turbulent Prandtl number is given already in the one-loop approximation and does not depend on $d$ while turbulent Prandt numbers in first two cases show very similar behaviour as functions of dimension $d$ in the two-loop approximation.
\end{abstract}

\section{Introduction}

The problems related to the behavior of various admixtures in turbulent environments are among the most studied in the framework of the turbulent fluid dynamics. Typical examples are scalar and vector impurities in given turbulent systems, e.g., the temperature in the problem of thermal diffusion or the problem of an impurity with internal structure in turbulent environments [1, 2], as well as the weak magnetic field in a turbulent plasma (electrically conductive turbulent fluid) described by the turbulent magnetohydrodynamics (MHD) [3, 4].

An effective approach for theoretical analysis of diffusion processes in fully developed turbulent environments is the field theoretic renormalization group (RG) technique (see, e.g., Ref. [5] and references cited therein). During the last few decades various field theoretical models of passive advection were developed for the analysis of the intermittency and anomalous scaling behavior of various correlation functions, for the investigation of deviations from the simple scaling predictions of the classical phenomenological Kolmogorov-Obukhov theory [6]. Properties of the anomalous scaling were reported in all these investigations.

On the other hand, one of the most important characteristics of the diffusion processes in fluids is the corresponding Prandtl number. The Prandtl number is defined as the ratio of the coefficient

\footnotetext{
$\star$ e-mail: jurcisine@ saske.sk

$\star \star$ e-mail: jurcisin@saske.sk

$\star \star \star$ e-mail: remecky@saske.sk
} 
of kinematic viscosity to the coefficient of diffusivity of the system. The Prandtl numbers usually strongly depend on the microscopic structure of the fluid when the value of the Reynolds number (Re) of the flow is low. The situation changes in the state of the so-called fully developed turbulence, i.e., where the fluid motion is characterized by very high Reynolds number (in the ideal case $\operatorname{Re} \rightarrow \infty$ ), when the Prandtl numbers reach universal values which are known as effective or turbulent Prandtl numbers.

In the present paper we investigate the turbulent vector Prandtl number in the framework of the general $\mathcal{A}$ model [7-10] of a passive vector quantity advected by the turbulent velocity field driven by the stochastic Navier-Stokes equation. This model describes three physically important cases of passive vector admixture, namely, the passive advection of the magnetic field in a conductive turbulent environment in the framework of the kinematic MHD turbulence with $\mathcal{A}=1[8,11,12]$, the passive admixture of a vector impurity by the Navier-Stokes turbulent flow with $\mathcal{A}=0[13,14]$, and the model of linearized Navier-Stokes equation with $\mathcal{A}=-1$.

\section{Mathematical Model of the Turbulent System}

In what follows, we consider the advection of a passive vector field $\mathbf{b} \equiv \mathbf{b}(x)$, where $x \equiv(t, \mathbf{x})$, driven by the incompressible Navier-Stokes velocity field $\mathbf{v}(x)$ described by the following system of stochastic equations:

$$
\begin{gathered}
\partial_{t} \mathbf{b}+(\mathbf{v} \cdot \partial) \mathbf{b}=u_{0} v_{0} \Delta \mathbf{b}+\mathcal{A}(\mathbf{b} \cdot \partial) \mathbf{v}-\partial \boldsymbol{Q}+\mathbf{f}^{\mathbf{b}}, \\
\partial_{t} \mathbf{v}+(\mathbf{v} \cdot \partial) \mathbf{v}=v_{0} \Delta \mathbf{v}-\partial \mathcal{P}+\mathbf{f}^{\mathbf{v}},
\end{gathered}
$$

where $v_{0}$ is viscosity coefficient, $u_{0}$ is dimensionless reciprocal general vector Prandtl number, $\mathcal{P}$ and $Q$ represent corresponding pressures, and the statistics of the random forces $\mathbf{f}^{\mathbf{b}}$ and $\mathbf{f}^{\mathbf{v}}$ are supposed Gaussian with zero means and correlation functions

$$
\begin{aligned}
& D_{i j}^{b}\left(x ; x^{\prime}\right) \equiv\left\langle f_{i}^{b}(x) f_{j}^{b}\left(x^{\prime}\right)\right\rangle=\delta\left(t-t^{\prime}\right) C_{i j}\left(\left|\mathbf{x}-\mathbf{x}^{\prime}\right| / L\right), \\
& D_{i j}^{v}\left(x, x^{\prime}\right) \equiv\left\langle f_{i}^{v}(x) f_{j}^{v}\left(x^{\prime}\right)\right\rangle=\delta\left(t-t^{\prime}\right) \int \frac{d^{d} \mathbf{k}}{(2 \pi)^{d}} g_{0} v_{0}^{3} k^{4-d-2 \varepsilon} P_{i j}(\mathbf{k}) e^{i \mathbf{k}\left(\mathbf{x}-\mathbf{x}^{\prime}\right)},
\end{aligned}
$$

where the explicit form of the function $C_{i j}$ is not important, $P_{i j}(\mathbf{k})=\delta_{i j}-k_{i} k_{j} / k^{2}$ is the ordinary transverse tensor projector, $g_{0}$ plays the role of the coupling constant of the model, and the physical value of formally small parameter $0<\varepsilon \leq 2$ is $\varepsilon=2$.

The stochastic model given by Eqs. (1)-(4) is equivalent to the field theoretic model of the double set of fields $\Phi=\left\{\mathbf{v}, \mathbf{b}, \mathbf{v}^{\prime}, \mathbf{b}^{\prime}\right\}$ with the action functional given as follows

$$
\begin{aligned}
S(\Phi)= & \frac{1}{2} \int d t_{1} d^{d} \mathbf{x}_{1} d t_{2} d^{d} \mathbf{x}_{2}\left[v_{i}^{\prime}\left(x_{1}\right) D_{i j}^{v}\left(x_{1} ; x_{2}\right) v_{j}^{\prime}\left(x_{2}\right)+b_{i}^{\prime}\left(x_{1}\right) D_{i j}^{b}\left(x_{1} ; x_{2}\right) b_{j}^{\prime}\left(x_{2}\right)\right] \\
& +\int d t d^{d} \mathbf{x}\left\{\mathbf{v}^{\prime}\left[-\partial_{t} \mathbf{v}+v_{0} \Delta \mathbf{v}-(\mathbf{v} \cdot \partial) \mathbf{v}\right]\right\} \\
& +\int d t d^{d} \mathbf{x}\left\{\mathbf{b}^{\prime}\left[-\partial_{t} \mathbf{b}+v_{0} u_{0} \Delta \mathbf{b}-(\mathbf{v} \cdot \partial) \mathbf{b}+\mathcal{A}(\mathbf{b} \cdot \partial) \mathbf{v}\right]\right\}
\end{aligned}
$$

The field theoretic model (5) corresponds to the standard Feynman diagrammatic perturbation theory which is used for the analysis of the original stochastic model. Note that the formulation of the stochastic problem given by Eqs. (1)-(4) through the field theoretic model with the action functional Eq. (5) allows one to use the well-defined field theoretic tools, e.g., the RG technique, to analyze the problem. The RG analysis of the field theoretic model described by the action functional (5) is based on analysis of UV divergences of the model. The detailed analysis is given in [15]. 


\section{Prandtl Number in the Two-Loop Approximation}

We are interested in the IR asymptotic scaling behavior of the correlation functions of the model deep inside the inertial interval. This behavior is driven by the corresponding IR stable fixed point of the RG equations. Coordinates of the fixed point $\left(g_{*}, u_{*}\right)$ deep inside the inertial interval are determined by the requirement of vanishing of the corresponding $\beta$ functions (see Ref. [15] for details).

The nontrivial IR stable fixed point, i.e., the IR stable fixed point with $g_{*} \neq 0$ and $u_{*} \neq 0$, in the two-loop approximation of the present model reads

$$
g_{*}=g_{*}^{(1)} \varepsilon+g_{*}^{(2)} \varepsilon^{2}+O\left(\varepsilon^{3}\right), \quad u_{*}=u_{*}^{(1)}+u_{*}^{(2)} \varepsilon+O\left(\varepsilon^{2}\right),
$$

where

$$
g_{*}^{(1)}=\frac{(2 \pi)^{2 d}}{S_{d}} \frac{8(d+2)}{3(d-1)}, \quad g_{*}^{(2)}=\frac{(2 \pi)^{2 d}}{S_{d}} \frac{8(d+2)}{3(d-1)} \lambda,
$$

and

$$
u_{*}^{(1)}=\frac{1}{3 a_{2}}\left[-2 a_{2}-\frac{2^{1 / 3} b_{1}}{\left(b_{2}+b_{3}\right)^{1 / 3}}+\frac{\left(b_{2}+b_{3}\right)^{1 / 3}}{2^{1 / 3}}\right], u_{*}^{(2)}=\frac{2(d+2)}{d\left[1+2 u_{*}^{(1)}\right]}\left[\lambda-\frac{128(d+2)^{2}}{3(d-1)^{2}} \mathcal{B}\left(u_{*}^{(1)}\right)\right],
$$

with the explicit forms of the coefficients $\mathcal{B}\left(u_{*}^{(1)}\right), \lambda$, and $a_{i}$ and $b_{i}$ for $i=1,2$ given in [15].

Using the same procedure as in Ref. [16] the corresponding two-loop formula for the turbulent inverse vector Prandtl number $u_{\text {eff }}$ in the framework of the general $\mathcal{A}$ model of passively advected vector quantity can be derived and written in the similar form as in Ref. [16], namely,

$$
u_{\mathrm{eff}}=u_{*}^{(1)}\left(1+\varepsilon\left\{\frac{1+u_{*}^{(1)}}{1+2 u_{*}^{(1)}}\left[\lambda-\frac{128(d+2)^{2}}{3(d-1)^{2}} \mathcal{B}\left(u_{*}^{(1)}\right)\right]+\frac{(2 \pi)^{d}}{S_{d}} \frac{8(d+2)}{3(d-1)}\left[a_{v}-a_{b}\left(u_{*}^{(1)}\right)\right]\right\}\right),
$$

where $a_{v}$ and $a_{b}\left(u_{*}^{(1)}\right)$ are given by the corresponding expansion to the leading order in $\varepsilon$ of the scaling functions of response functions $\left\langle b b^{\prime}\right\rangle$ and $\left\langle v v^{\prime}\right\rangle$ of the advected vector and velocity field, respectively. For more details and the explicit expressions for the aforementioned quantities see [15].

The turbulent vector Prandtl number in the two-loop approximation is given as the inverse value of the effective inverse Prantl number, i.e.,

$$
\operatorname{Pr}_{\mathcal{A}, \mathrm{t}}=1 / u_{\mathrm{eff}} .
$$

\section{Discussion}

In the present work we devoted special attention to the three important and physically interesting cases of the general model of vector advection, namely, to the model of the linearized Navier-Stokes equation $\mathcal{A}=-1$, to the passive admixture of vector impurity by Navier-Stokes turbulent flow $\mathcal{A}=0$ and to the model of passive advection of the magnetic field in the framework of kinematic MHD turbulence $\mathcal{A}=1$.

It is shown that the turbulent Prandtl number $\operatorname{Pr}_{1, t}$ in the model of linearized Navier-Stokes equation $\left(\mathcal{A}=-1\right.$ ) is exactly given already in the one-loop approximation, $\operatorname{Pr}_{1,1}^{(1)}=\operatorname{Pr}_{1, t}$, see Fig. 1. Higher loop approximations vanish and the numerical value is equal to 1 for all values of the spatial dimension $d>2$. The nontrivial conclusion is that in this model, coefficients of turbulent diffusivity and the turbulent viscosity are numerically equal.

On the other hand, the two-loop turbulent Prandtl numbers $\operatorname{Pr}_{\mathrm{m}, \mathrm{t}}(\mathcal{A}=1)$ and $\operatorname{Pr}_{\mathrm{v}, \mathrm{t}}(\mathcal{A}=0)$ show very similar behaviours as functions of the spatial dimension $d$, see Fig. 1. They are both increasing functions of $d$ and both go to 1 in the limit $d \rightarrow \infty$. 


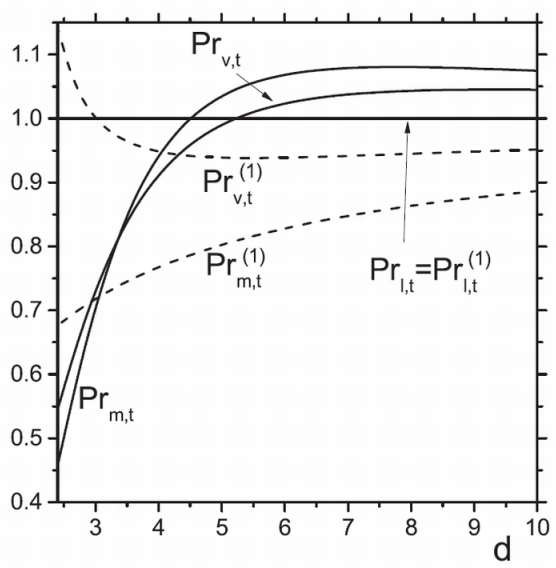

Figure 1. The behavior of the two-loop turbulent Prandtl numbers in the general $\mathcal{A}$ model for three special cases, namely for $\mathcal{A}=-1\left(\operatorname{Pr}_{1, t}\right), \mathcal{A}=0\left(\operatorname{Pr}_{\mathrm{v}, \mathrm{t}}\right)$ and for $\mathcal{A}=1\left(\operatorname{Pr}_{\mathrm{m}, \mathrm{t}}\right)$. The corresponding one-loop curves $\operatorname{Pr}_{\ldots}^{(1)}$ are also shown for comparison.

\section{Acknowledgements}

The work was supported by the VEGA grant No. 2/0065/17.

\section{References}

[1] A.S. Monin and A.M. Yaglom, Statistical Fluid Mechanics, Vol. 2 (Cambridge, MA, MIT Press, 1975)

[2] W.D. McComb, The Physics of Fluid Turbulence (Oxford, Clarendon, 1990)

[3] A. Yoshizawa, S.I. Itoh, and K. Itoh, Plasma and Fluid Turbulence: Theory and Modelling (Bristol, IoP, 2003)

[4] D. Biskamp, Magnetohydrodynamic Turbulence (Cambridge, Cambridge University Press, 2003)

[5] A.N. Vasil'ev, The Field Theoretic Renormalization Group in Critical Behavior Theory and Stochastic Dynamics (Chapman \& Hall/CRC, CRC Press, 2004)

[6] A.N. Kolmogorov, Dokl. Akad. Nauk SSSR 30, 301 (1941)

[7] L.Ts. Adzhemyan, N.V. Antonov, A. Mazzino, P. Muratore-Ginanneschi, and A.V. Runov, Europhys. Lett. 55, 801 (2001)

[8] N.V. Antonov, M. Hnatich, J. Honkonen, and M. Jurčišin, Phys. Rev. E 68, 046306 (2003)

[9] H. Arponen, Phys. Rev. E 79, 056303 (2009)

[10] N.V. Antonov and N.M. Gulitskiy, Theor. Math. Phys. 176, 851 (2013)

[11] E. Jurčišinová, M. Jurčišin, and R. Remecký, Phys. Rev. E 84, 046311 (2011)

[12] E. Jurčišinová, M. Jurčišin, R. Remecký, and P. Zalom, Phys. Rev. E 87, 043010 (2013)

[13] E. Jurčišinová, M. Jurčišin, and R. Remecký, Phys. Rev. E 88, 011002(R) (2013)

[14] E. Jurčišinová, M. Jurčišin, and P. Zalom, Phys. Rev. E 89, 043023 (2014)

[15] E. Jurčišinová, M. Jurčišin, and R. Remecký, Phys. Rev. E 93, 033106 (2016)

[16] L.Ts. Adzhemyan, J. Honkonen, T.L. Kim, and L. Sladkoff, Phys. Rev. E 71, 056311 (2005) 